\title{
BP-OP-1-5
}

\section{Changes in total lymphocyte count and neutrophil-to-lymphocyte ratio after curative pancreatectomy in patients with pancreas adenocarcinoma and their prognostic role}

\author{
Eun Young KIM', Tae Ho HONG*2
}

'Division of Trauma and Surgical Critical Care, Department of Surgery, Seoul St. Mary's Hospital, The Catholic University of Korea, Seoul, Korea ${ }^{2}$ Division of Hepato-Biliary and Pancreas Surgery, Department of Surgery, Seoul St. Mary's Hospital, The Catholic University of Korea, Seoul, Korea

Introduction: To assess the prognostic significance of postoperative changes in immune status represented by total lymphocyte count (TLC) and neutrophil-to-lymphocyte ratio (NLR) in resectable pancreatic cancer.

Methods: Patients who underwent curative pancreatectomy for pancreatic adenocarcinoma were divided into high and low groups according to cut-off values of TLC, and NLR measured preoperatively, immediately after surgery, and 1- or 6-month after surgery. Oncologic outcomes were compared between the two groups at different times, and prognostic roles of TLC and NLR were evaluated. Results: Of 193 patients, the median follow-up time was 22 months, and median survival was 18 months. Their immunologic status deteriorated within 3-4 days after the operation and recovered after that. At 1- and 6-month postoperatively, overall survival rates were significantly lower in the group with high NLR $(>2.535$ and $>3.21$, respectively) and low TLC $(<1.66 \times 109 / \mathrm{L}$ and $<1.62 \times 109 / \mathrm{L}$, respectively). In multiple regression analysis, elevated NLR at postoperative 1- and 6-month and decreased TLC at postoperative 1-month were significant prognosis predictors.

Conclusions: Changes in immune status such as decreased TLC and elevated NLR at postoperative 1- and 6-month are effective prognostic predictors after curative pancreatectomy in patients with pancreatic adenocarcinoma. 